\title{
Lifetimes of long-lived states in inhomogeneous magnetic fields
}

\author{
Maninder Singh ${ }^{\mathrm{a}, \mathrm{b}}$, Srinivas Chinthalapalli ${ }^{\mathrm{b}, \mathrm{c}, *}$, Geoffrey Bodenhausen ${ }^{\mathrm{b}, \mathrm{d}, \mathrm{e}, \mathrm{f}}$ \\ a Department of Chemistry, Indian Institute of Technology, Hauz Khas, 110016, New Delhi, India \\ ${ }^{\mathrm{b}}$ Institut des Sciences et Ingénierie Chimiques, Ecole Polytechnique Fédérale de Lausanne, CH-1015 Lausanne, Switzerland \\ ${ }^{c}$ Department of Chemistry, School of Chemical Sciences, Central University of Karnataka, Gulbarga, 585311, Karnataka, India \\ d École Normale Supérieure-PSL Research University, Département de Chimie, 24 rue Lhomond, F-75005 Paris, France \\ e Sorbonne Universités, UPMC Univ Paris 06, 4 Paris, France \\ ${ }^{\mathrm{f}}$ CNRS, UMR 7203 LBM, F-75005 Paris, France
}

\section{A R T I C L E I N F O}

Article history:

Received 2 November 2014

In final form 27 January 2015

Available online 3 February 2015

\begin{abstract}
A B S T R A C T
Long-lived states (LLS), also known as singlet states, have been widely studied in the last decade. So far, LLS have only been observed in homogeneous magnetic fields, which preclude applications to many biological samples that are inherently inhomogeneous. We present a method to measure the lifetimes $T_{L L S}$ of long-lived states in inhomogeneous magnetic fields, which combines established sequences for the excitation of LLS with their conversion into long-lived coherences (LLC) that can be detected by windowed acquisition. The method is applied to a pair of diastereotopic scalar-coupled protons of glycine in the dipeptide Alanine-Glycine (Ala-Gly).
\end{abstract}

(c) 2015 The Authors. Published by Elsevier B.V. This is an open access article under the CC BY-NC-ND license (http://creativecommons.org/licenses/by-nc-nd/4.0/).

\section{Introduction}

If the static magnetic field is not perfectly homogeneous, NMR signals suffer from inhomogeneous broadening that makes it difficult to determine spectral parameters like chemical shifts, coupling constants, and the underlying homogeneous linewidths. It is possible to extract high resolution NMR information by refocusing inhomogeneous line broadening using some form of spin-echo spectroscopy [1], but the task of recovering the full spectral information often remains an elusive objective.

Recently, long-lived states (LLS) have attracted considerable attention because of their long spin memory [2]. If we consider two coupled protons, it is easy to prepare LLS by a simple sequence of pulses such as described by Sarkar et al. [3]. The population of the resulting LLS can then be 'sustained' using sequences of radio-frequency pulses with or without windows [4,5]. The difference in the decay rates of the populations of LLS and ordinary Zeeman states is due to the formers' immunity to the dominant intramolecular dipole-dipole relaxation mechanism. LLS can be utilized to study various slow dynamical processes that are normally difficult to characterize because of short longitudinal relaxation times [3]. LLS are not necessarily confined to pairs of coupled

Abbreviations: LLC, Long-lived coherences; LLS, Long-lived states; SID, Sustained induction decay; SQC, Single quantum coherence.

* Corresponding author at: Department of Chemistry, School of Chemical Sciences, Central University of Karnataka, Gulbarga, 585311 Karnataka, India.

E-mail address: cnuvas2006@gmail.com (S. Chinthalapalli). protons, but can also be excited in multi-spin systems, as occur in many molecules of biological importance [6-10]. Furthermore, LLS signals can be enhanced by dissolution dynamic nuclear polarization (DNP) [11], thus allowing the study of substrates in very low concentrations [12]. Although applications of LLS are increasingly popular in various fields of chemistry and biology, a major challenge is their detection in inhomogeneous magnetic fields. Since LLS are not directly observable, they are usually converted into detectable single-quantum coherences that are sensitive to magnetic field inhomogeneities. Thus the application of LLS is challenging when the magnetic field homogeneity is poor as for in-cellulo or in vivo NMR.

Zero-quantum coherences (ZQCs) between the singlet and central triplet states are known as long-lived coherences (LLCs) [13-15]. Their lifetimes $T_{L L C}$ can be much longer than those of single quantum coherences (SQCs), but shorter than those of LLS, since LLCs are not immune to intramolecular dipole-dipole relaxation. We have shown that LLCs are immune to spatial and temporal variations of external static magnetic fields, so that they can provide spectra with very high resolution even in moderately inhomogeneous fields [16]. We have also shown that LLCs can be used to record high-resolution two-dimensional NMR spectra in inhomogeneous magnetic fields to determine scalar couplings and differences of chemical shifts [17]. However, studies in inhomogeneous magnetic fields have not been extended from LLCs to the realm of LLS so far.

The lifetime $T_{L L S}$ can normally be determined after conversion into SQCs by monitoring the latters' signal intensities as a 


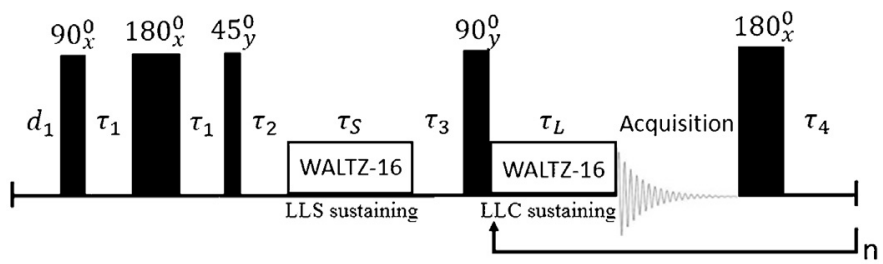

Figure 1. Pulse sequence designed to determine $T_{L L S}$ in inhomogeneous magnetic fields. Typically, the total number of loops may be $n=1024$, and the recovery delay $d_{1}$ may be $3-5$ times $T_{1}, \tau_{1}=1 /(4 J), \tau_{2}=1 /\left(2 \Delta v_{I S}\right), \tau_{3}=1 /\left(4 \Delta v_{I S}\right)$, where $J$ is the scalar coupling constant and $\Delta v_{I S}$ is the chemical shift difference of the two involved spins, $\tau_{S}$ is a variable delay where the LLS are sustained, $\tau_{L}$ is a fixed delay where the LLC are sustained (in our example $24 \mathrm{~ms}$ ), the acquisition time and $\tau_{4}$ have the same duration of $492.35 \mu \mathrm{s}$. All 246 points acquired at intervals of $2 \mu$ s within each acquisition block are averaged to make up a single point of the sustained induction decay (SID) [16]. The sustaining or spin locking pulses can be modulated in the manner of WALTZ-16 sequences.

function of the interval $\tau_{\mathrm{S}}$ where the LLS are sustained, and fitting their decay to an exponential function $\exp \left\{-\tau_{\mathrm{S}} / T_{L L S}\right\}[18,19]$. Unfortunately, this method is limited to homogeneous magnetic fields. We demonstrate here the measurement of LLS lifetimes in inhomogeneous magnetic fields by converting the LLS for detection into LLC that are insensitive to inhomogeneties. This can be achieved by combining sequences for the excitation and sustaining of LLS with sequences for the detection of LLC, as shown in Figure 1.

\section{Experimental}

All experiments were performed at room temperature using an ultra-shielded $500 \mathrm{MHz}$ Avance Bruker spectrometer equipped with an inverse $5 \mathrm{~mm}$ cryoprobe. A $0.5 \mathrm{M}$ solution of Ala-Gly was prepared in $0.5 \mathrm{ml} \mathrm{D}_{2} \mathrm{O}$. The chemical shift difference of the two diastereotopic protons of Gly is $\Delta v_{I S}=57 \mathrm{~Hz}$ and the scalar coupling constant is $J=-17.24 \mathrm{~Hz}$. The longitudinal relaxation time of these protons is $T_{1}=1.2 \mathrm{~s}$ and the transverse relaxation time of their SQCs is $T_{2} \approx 0.9 \mathrm{~s}$. The LLC sustaining time $\tau_{L}$ and the acquisition time (which must be equal to the refocusing interval $\tau_{4}$ ) depend on the spectral width in the $\omega_{2}$ domain. For the detection of LLC's in inhomogeneous fields, we chose a spectral width of $40 \mathrm{~Hz}$, hence a duration of the detection loop $\tau_{\text {loop }}=1 / 40 \mathrm{~Hz}=25 \mathrm{~ms}$ (this plays the role of a dwell time for the detection of LLC's), a spin-locking interval where the LLC are sustained by rf irradiation $\tau_{L}=24 \mathrm{~ms}$, and a refocusing pulse length $\tau_{\pi}=15.3 \mu \mathrm{s}$. The acquisition time and the refocusing interval must have the same duration and obey the relation $\tau_{4}=(1 / 2)\left[\tau_{\text {loop }}-\tau_{L}-\tau_{\pi}\right]=492.35 \mu \mathrm{s}$. The amplitude of the WALTZ-16 irradiation applied in both intervals $\tau_{S}$ and $\tau_{L}$ where the LLS and LLC are sustained was $1 \mathrm{kHz}$. Each LLC spectrum was acquired with $n=1024$ data points. A total of 13 experiments were carried out while incrementing the sustaining interval $\tau_{s}$ to monitor the decays of the integrals of the LLC signals which were fitted to an exponential function $\exp \left\{-\tau_{\mathrm{S}} / T_{L L S}\right\}$.

\section{Methods, results and discussion}

The pulse sequence consists of three parts (Figure 1): (i) broadband excitation and sustaining of LLS as described by Sarkar et al. [3]; (ii) conversion of LLS into LLC, and (iii) acquisition of signals derived from LLS as shown by Bornet et al. [16]. As seen from the Figure 1 , starting from equilibrium magnetization $\left(I_{z}+S_{z}\right)$, the initial $90_{x}^{\circ}$ pulse is followed by a spin echo sequence to create terms $\left(2 I_{x} S_{z}+2 I_{z} S_{x}\right)$ due to the evolution under the scalar coupling $J_{I S}$, regardless of the homogeneity of the static magnetic field. At the top of the echo, a $45_{y}^{\circ}$ pulse converts $\left(2 I_{x} S_{z}+2 I_{z} S_{x}\right)$ into $\left(2 I_{x} S_{x}-2 I_{z} S_{z}\right) \equiv\left(Z Q_{x}+D Q_{x}-2 I_{z} S_{z}\right)$, i.e., into a superposition of multiple-quantum coherences and zz order. The $\left(Z Q_{x}-2 I_{z} S_{z}\right)$ terms are converted into $\left(-Z Q_{x}-2 I_{z} S_{z}\right)$ under free evolution in the interval $\tau_{2}=1 /\left(2 \Delta v_{I S}\right)$, since both are immune to the inhomogeneity of the static field, whereas $D Q_{x}$ is dephased. The terms $Z Q_{x}$ and $2 I_{z} S_{z}$ are then sustained by a WALTZ-16 sequence for a variable time $\tau_{s}$. This $r f$ irradiation has the effect of suppressing the chemical shifts to that the spins $I$ and $S$ become magnetically equivalent. In the process, the $\left(-Z Q_{x}-2 I_{z} S_{z}\right)$ terms are transformed into a long-lived state (LLS), also known as "triplet-singlet imbalance" or "T/S imbalance", in analogy to "A/E imbalance" in methyl groups [20]. This amounts to a non-vanishing difference between the average population of the three triplet states that are symmetric under spin permutation and the population of the antisymmetric singlet state:

$$
\overline{|T\rangle\langle T|}-\overline{|S\rangle\langle S|}=\frac{4}{3}\left\langle I_{1} \cdot I_{2}\right\rangle \neq 0
$$

where $\overline{|T\rangle\langle T|}=1 / 3\left[\left|T_{+1}\right\rangle\left\langle T_{+1}|+| T_{0}\right\rangle\left\langle T_{0}|+| T_{-1}\right\rangle\left\langle T_{-1}\right|\right]$ and $\overline{|S\rangle\langle S|}=\left[\left|S_{0}\right\rangle\left\langle S_{0}\right|\right]$.

After the sustaining interval $\tau_{s}$, the rf field is switched off, so that the LLS is converted back into $\left(-Z Q_{x}-2 I_{z} S_{Z}\right)$ terms. The delay $\tau_{S}=1 /\left(2 \Delta v_{I S}\right)$ then allows the conversion of $\left(-2 I_{x} S_{x}-2 I_{y} S_{y}-2 I_{z} S_{z}\right) \equiv\left(-Z Q_{x}-2 I_{z} S_{z}\right) \quad$ into $\left(2 I_{x} S_{y}-2 I_{y} S_{x}-2 I_{z} S_{z}\right) \equiv\left(-Z Q_{y}-2 I_{z} S_{z}\right)$. Again, neither $Z Q_{y}$ nor zz-order are affected by the magnetic field inhomogeneity. Now a $90_{y}^{0}$ pulse transforms $\left(-Z Q_{y}-2 I_{z} S_{z}\right)$ into $\left(-2 I_{y} S_{z}+2 I_{z} S_{y}-2 I_{z} S_{z}\right)$, that is, into anti-phase SQC terms in addition to unobservable zz-order. These anti-phase terms, if one attempted to detect them in an inhomogeneous static field, would be obscured by the linewidth. In order to alleviate this problem, the signal has to be acquired by some procedure that is not influenced by static field inhomogeneities. One such method is to convert the order from LLS to LLC, and then to detect the signal using an "on the fly" or "windowed" LLC acquisition scheme which is immune to static field inhomogenities.

In the product operator formalism, an LLC can be represented by terms $\left(I_{x}-S_{x}\right)$ or $\left(2 I_{y} S_{z}-2 I_{z} S_{y}\right)$ which slowly decay when they are spin-locked [15]. The state $\left(-2 I_{y} S_{z}+2 I_{z} S_{y}-2 I_{z} S_{z}\right)$ remaining after the $90_{y}^{\circ}$ pulse contains anti-phase SQC terms which can be observed in the form of narrow spectral lines if acquired by spin locking alternating with windowed acquisition. In the observation windows, there is a partial conversion under the effect of the scalar coupling $J_{I S}$ so that the Fourier transformation of the signal detected in the $t_{2}$ interval gives rise to a doublet at $+2 \pi J_{I S}$ and $-2 \pi J_{I S}$ in the $\omega_{2}$ dimension of which only one peak is shown here.

In order to measure the relaxation time $T_{L L S}$, the duration of the sustaining interval $\tau_{s}$ has to be varied while detecting the corresponding LLC signals (Figure 3). In this way we can simultaneously measure LLS as well as LLC lifetimes. The $T_{L L S}$ value can be obtained by fitting the decaying signals to an exponential function $\exp \left\{-\tau_{S} / T_{L L S}\right\}$ (Figure 4). The LLC lifetime is obtained from $T_{L L C}=\left(\pi \Delta v_{L L C}\right)^{-1}$ where $\Delta v_{L L C}$ is the linewidth at half height of the LLC signal in the $\omega_{2}$ dimension, expressed in $\mathrm{Hz}$.

In high resolution solution-state NMR, the required magnetic field homogeneity must typically be on the order of $10^{-9}$ or $1 \mathrm{ppb}$ [21]. In our experiments, we deliberately made the magnetic field inhomogeneous by missetting various shim currents $\left(z_{1}, z_{2}, z_{3}, x\right.$, $\left.y, z_{0} x, z_{0} y\right)$ to broaden the linewidth as shown in Figure 2 for a homogeneous field with a linewidth $\Delta v=1.8 \mathrm{~Hz}$ (red line) and for an inhomogeneous field with a linewidth $\Delta v=17 \mathrm{~Hz}$ (blue line). Such changes in the homogeneity of the static magnetic field have little effect on the linewidths of LLCs [16]. 


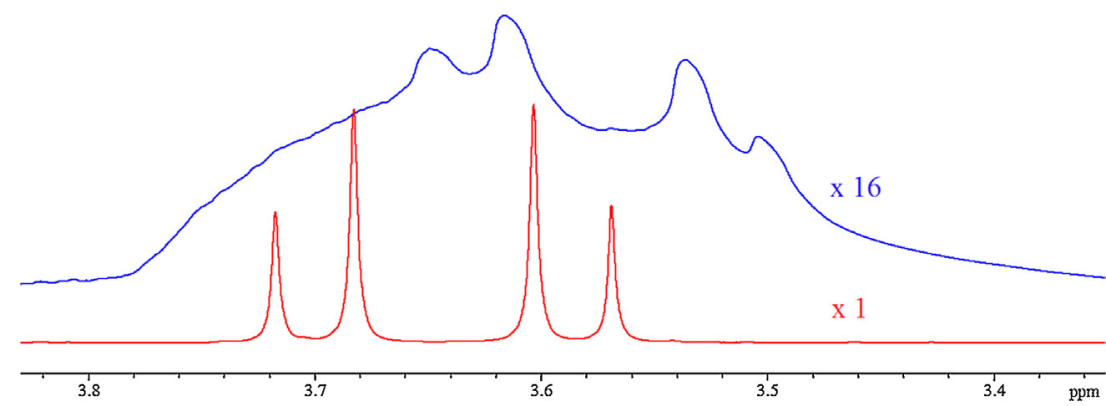

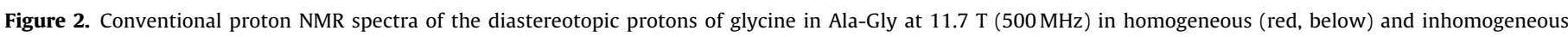
magnetic fields (blue, above). (For interpretation of the references to color in this figure legend, the reader is referred to the web version of this article.)
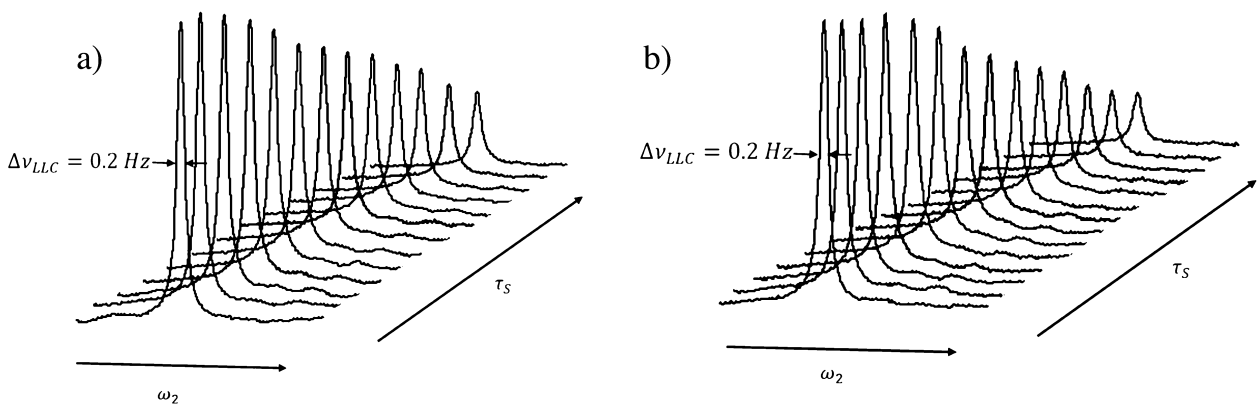

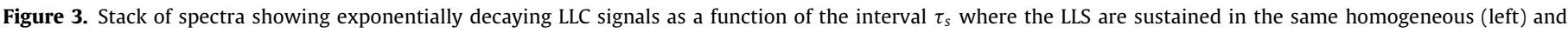
inhomogeneous (right) magnetic fields as in Figure 2.

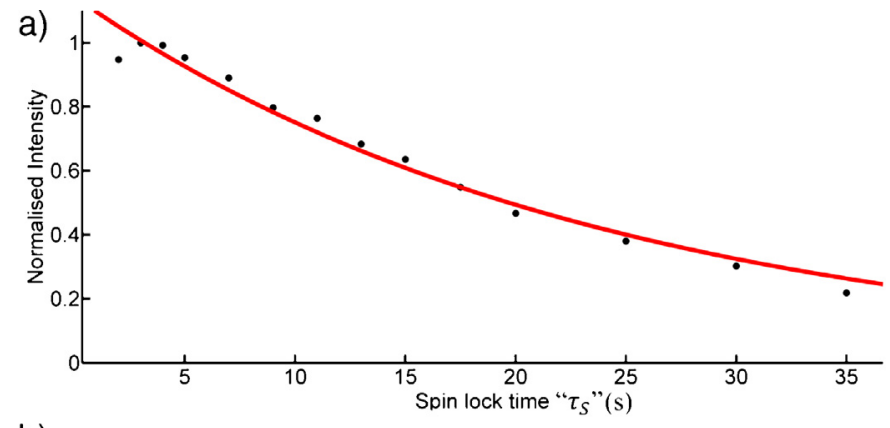

b)

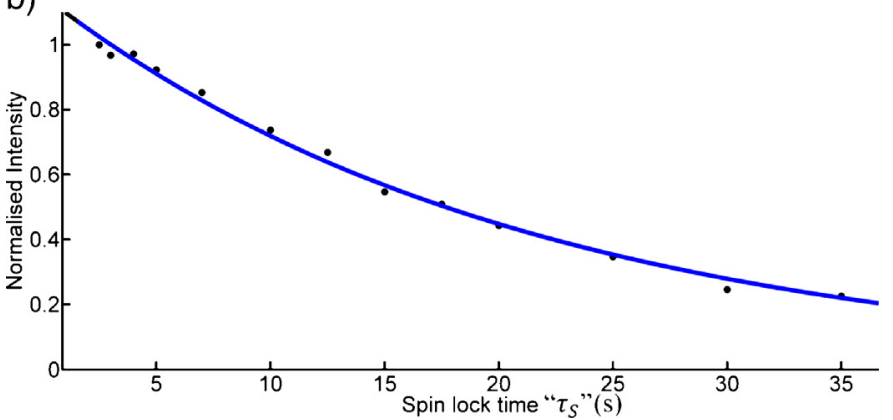

Figure 4. Experimental normalized LLC signal intensities (black dots) as a function of the interval where the LLS are sustained $0<\tau_{s}<35 \mathrm{~s}$ with exponential fits in a homogeneous magnetic field ( $T_{L L S}=22.4 \pm 0.70 \mathrm{~s}$, red line, top) and in an inhomogeneous magnetic field ( $T_{L L S}=21.1 \pm 0.6 \mathrm{~s}$, blue line, below). (For interpretation of the references to color in this figure legend, the reader is referred to the web version of this article.)

We have tested this pulse sequence on the two scalar-coupled diastereotopic protons of glycine in the dipeptide Ala-Gly. The observations were made both in homogeneous and inhomogeneous static magnetic fields (Figs. 3 and 4). The small deviations from exponential behavior in the initial parts of the decay curves are believed to be due to the equilibration of the populations within the triplet manifold $\left\{\left|T_{1}\right\rangle,\left|T_{0}\right\rangle,\left|T_{-1}\right\rangle\right\}$ [22]. The lifetimes $T_{L L S}$ obtained are the same within experimental error, that is, $T_{L L S}=22.4 \pm 0.7$ and $21.1 \pm 0.6 \mathrm{~s}$ in homogeneous and inhomogeneous fields, respectively. The linewidths of the LLC signals remained approximately the same throughout. The ability to mask inhomogeneities is directly proportional to the strength of rf field used for sustaining the LLS and LLC, as may be shown by simulations using the GAMMA platform. For larger rf field strengths, the sequence works even when the inhomogeneities are large, albeit at the cost of rf heating. LLCs are more prone to suffer from inhomogeneities than LLS.

\section{Concluding remarks}

In summary, we conclude that one can determine the lifetime $T_{L L S}$ of LLS even when the static field is inhomogeneous. The $T_{L L S}$ values obtained in both homogeneous and inhomogeneous static magnetic field are consistent. The method may find applications in measuring the lifetimes of LLS in-cellulo, in vivo, in gas phase, and in other systems where the homogeneity of the static field is compromised.

\section{Acknowledgements}

The authors are indebted to Aurélien Bornet and Diego Carnevale for stimulating suggestions. They would like to thank the Indian Institute of Technology Delhi (IITD) and the École polytechnique fédérale de Lausanne (EPFL) for providing opportunities to work under the IITD-EPFL Exchange Program at EPFL, Lausanne, Switzerland. This work was supported by the Swiss National Science Foundation (SNSF), the Swiss Commission for Technology and Innovation (CTI) and the European Research Council (Grant no. 339754) (ERC, contract 'para-water'). We thank Prof. Narayanan D Kurur for helpful comments and discussions. 


\section{References}

[1] (a) R. Freeman, H.D.W. Hill, J. Chem. Phys. 54 (1971) 301; (b) D. Hall, T.J. Norwood, Chem. Commun. (1986) 1508.

[2] M.H. Levitt, Annu. Rev. Phys. Chem. 63 (2012) 89.

[3] R. Sarkar, P.R. Vasos, G. Bodenhausen, J. Am. Chem. Soc. 129 (2007) 328.

[4] S.S. Roy, T.S. Mahesh, G.S. Agarwal, Phys. Rev. A 83 (2011) 062326.

[5] R. Sarkar, P. Ahuja, D. Moskau, P.R. Vasos, G. Bodenhausen, ChemPhysChem 8 (2007) 2652.

[6] G. Pileio, M. Concistrè, M. Carravetta, M.H. Levitt, J. Magn. Reson. 182 (2006) 353.

77] G. Pileio, M.H. Levitt, J. Magn. Reson. 187 (2007) 141.

[8] A.K. Grant, E. Vinogradov, J. Magn. Reson. 193 (2008) 177.

[9] E. Vinogradov, A.K. Grant, J. Magn. Reson. 194 (2008) 46.

[10] P. Ahuja, R. Sarkar, P.R. Vasos, G. Bodenhausen, ChemPhysChem 10 (2009) 2217.

[11] J.H. Ardenkjaer-Larsen, B. Fridlund, A. Gram, G. Hansson, L. Hansson, M.H. Lerche, R. Servin, M. Thaning, K. Golman, Proc. Natl. Acad. Sci. U.S.A. 100 (2003) 10158.
[12] P.R. Vasos, A. Comment, R. Sarkar, P. Ahuja, S. Jannin, J.P. Ansermet, J.A. Konter, P. Hautle, B.V. Brandt, G. Bodenhausen, Proc. Natl. Acad. Sci. U.S.A. 106 (2009) 18469.

[13] G. Pileio, M. Carravetta, M.H. Levitt, Phys, Rev. Lett. 103 (2009) 083002.

[14] R. Sarkar, P. Ahuja, P.R. Vasos, G. Bodenhausen, Phys. Rev. Lett. 104 (2010) 053001.

[15] R. Sarkar, P. Ahuja, P.R. Vasos, A. Bornet, O. Wagnières, G. Bodenhausen, Prog. Nucl. Magn. Reson. Spectrosc. 59 (2011) 83.

[16] A. Bornet, S. Jannin, J.A. Konter, P. Hautle, B. van den Brandt, G. Bodenhausen, J. Am. Chem. Soc. 133 (2011) 15644.

[17] S. Chinthalapalli, A. Bornet, T.F. Segawa, R. Sarkar, S. Jannin, G. Bodenhausen, Phys. Rev. Lett. 109 (2012) 047602.

[18] M. Carravetta, O.G. Johannessen, M.H. Levitt, Phys. Rev. Lett. 94 (2004) 153003

[19] M. Carravetta, M.H. Levitt, J. Am. Chem. Soc. 126 (2004) 6228.

[20] B. Meier, J.N. Dumez, G. Stevanato, J.T. Hill-Cousins, S.S. Roy, P. Hakansson, S. Mamone, R.C.D. Brown, G. Pileio, M.H. Levitt, J. Am. Chem. Soc. 135 (2013) 18746.

[21] Z. Chen, T. Hou, Z.W. Chen, D.W. Hwang, L.P. Hwang, Chem. Phys. Lett. 386 (2004) 200.

[22] S.S. Roy, T.S. Mahesh, J. Magn. Reson. 206 (2010) 127. 